\title{
Neofidia Strother, a new name for Fidia Baly, 1863 and redescription of Fidia kanaraensis (Jacoby, 1895) with a new host record and notes on natural history (Coleoptera, Chrysomelidae, Eumolpinae)
}

\author{
S. Amritha KUMARI ${ }^{1}$, A.G. MOSEYKO ${ }^{2}$, M.S. STROTHER ${ }^{3}$ \& K.D. PRATHAPAN ${ }^{4, *}$ \\ ${ }^{1}$ Department of Agricultural Entomology, Kerala Agricultural University, \\ Vellayani P. O., Trivandrum-695522, Kerala, India. \\ ${ }^{2}$ Laboratory of Insect Systematics, Zoological Institute, Russian Academy of Sciences, \\ Universitetskaya nab. 1, 199034 St Petersburg, Russia. \\ ${ }^{3} 12911120^{\text {th }}$ Ave NE, G-100, Kirkland, WA 98034, USA. \\ ${ }^{4}$ Department of Agricultural Entomology, Kerala Agricultural University, \\ Vellayani P. O., Trivandrum-695522, Kerala, India. \\ *Corresponding author: prathapankd@gmail.com \\ ${ }^{1}$ Email: amritha2005kau@gmail.com \\ 2Email: chrysolesha@mail.ru \\ 32Email: msean68@gmail.com

\footnotetext{
${ }^{1}$ urn:1sid:zoobank.org:author:68EB2530-5202-46F6-BA94-44B9D6CF09B8

${ }^{2}$ urn:1sid:zoobank.org:author:3017821E-5745-48E8-8CB3-C8A9AC5662BF

${ }^{3}$ urn:1sid:zoobank.org:author:1EF70328-9AC6-4CC8-A175-B79133CC1087

${ }^{4}$ urn:Isid:zoobank.org:author:68E05D80-9F21-4632-8AEE-92F3994CBEE0
}

\begin{abstract}
Neofidia Strother nom. nov., is proposed as the replacement name for Fidia Baly, 1863, a junior homonym of Fidia Motschulsky, 1861 (not 1860, Griffin 1936). A list of the included species of Neofidia Strother nom. nov. and Fidia Motschulsky, 1861 is provided for clarity. Fidia medvedevi nom. nov. is the new replacement name proposed for Lypesthes vietnamicus Medvedev, 2015. Fidia kanaraensis (Jacoby, 1895) is redescribed and habitus, male and female genitalia are figured. Cashew (Anacardium occidentale L.) is reported as a new host of $F$. kanaraensis and partial information on the life history is provided. Eggs are laid singly on the surface of soil, and are covered with excreta and soil. Larvae tunnel into the tender roots. Adults are nocturnal and feed on tender leaves.
\end{abstract}

Keywords. India, leaf beetles, new combinations, endophallus, female genitalia.

Kumari S.A., Moseyko A.G., Strother M.S. \& Prathapan K.D. 2020. Neofidia Strother, a new name for Fidia Baly, 1863 and redescription of Fidia kanaraensis (Jacoby, 1895) with a new host record and notes on natural history (Coleoptera, Chrysomelidae, Eumolpinae). European Journal of Taxonomy 654: 1-25.

https://doi.org/10.5852/ejt.2020.654

\section{Introduction}

The genus group name Fidia, coined by Dejean (1836), remained unavailable till Motschulsky (1861) described Fidia atra from the Old World, as Dejean (1836) had merely listed two New World species, 
F. lurida Dejean, 1836 and F. murina Dejean, 1836, without a description, definition or indication. Motschulsky's species description, in combination with the generic name Fidia, unwittingly made this generic group name available. Baly (1863) described the Old World genera Leprotes (type species: Adoxus gracilicornis Baly, 1861 by original designation) as well as Lypesthes (type species: Fidia atra Motschulsky, 1861 by original designation), provided a description of Fidia Dejean, 1836 and listed the New World Fidia lurida Dejean, 1836 as its type species. Baly's description made both the names Fidia and Fidia lurida available, in effect making Fidia Baly, 1863, a junior homonym of Fidia Motschulsky, 1861. Chen (1935) treated Leprotes Baly, 1863 as a junior synonym of Lypesthes Baly, 1863. Following Baly (1863) and Chen (1935), the name Lypesthes has been applied consistently for the Old World genus based on the type species Fidia atra Motschulsky, 1861. Similarly, Fidia Baly, 1863 has been applied to the New World genus group established on the type species Fidia lurida Baly. Strother \& Staines (2008) reviewed the New World Fidia Baly, 1863 and provided an account of the nomenclatural history of the generic name. Strother et al. (2007) applied to the ICZN for suppression of the name Fidia Motschulsky, 1861 (not 1860, Griffin 1936) and conservation of the usage of Lypesthes Baly, 1863. The Commission, however, rejected this proposal and upheld the priority of Fidia Motschulsky, 1861 (ICZN 2009). Löbl (2010) mentioned this issue, but did not make the nomenclatural changes. The Old World genus Fidia Motschulsky, 1861 is distinct from the New World Fidia Baly, 1863. Following the ruling of the Commission, reaffirming the priority of Fidia Motschulsky, 1861 the junior homonym Fidia Baly, 1863 is renamed here, as no available junior synonym exists for Fidia Baly, 1863.

Fidia Motschulsky comprises 34 species distributed in the Oriental and Palearctic Regions (Jacoby 1908; Pic 1923, 1924, 1928; Chen 1935; Eroshkina 1992; Medvedev \& Zoia 1996; Moseyko \& SprecherUebersax 2010).

Information on the biology of Fidia Motschulsky is limited. Isono (1988) stated that the eggs are covered entirely with excrement in Lypesthes. Lee (2012) described the larva of F. atra. According to Jolivet et al. (2014), the coating of eggs or egg masses with glandular secretions and/or faeces is known in a number of genera including Lypesthes. A number of host plants is recorded for species of the genus (Table 1).

Fidia kanaraensis (Jacoby, 1895) comb. nov. was described originally by Jacoby (1895) in the genus Leprotes, based on the specimens from Mahe and the erstwhile Kanara in south India. Jacoby (1908) treated it under the Fauna of British India and extended its known range of distribution to the erstwhile southern Bombay and Bengal. No further information on its morphology, genitalia or natural history is known, except that it was listed as a pest of mango (Mangifera indica L.) by Rajendran \& Singh (2016). The species is here redescribed with detailed information on its external morphology and genitalia. An attempt was made to elucidate its life cycle, that however, could not be completed successfully in the laboratory.

\section{Material and methods}

Specimens of Fidia kanaraensis were collected from Cardamom Research Station, Pampadumpara, Idukki ( $\left.9^{\circ} 48^{\prime} 23.7^{\prime \prime} \mathrm{N}, 77^{\circ} 10.04^{\prime} 9^{\prime \prime} \mathrm{E}\right)$; College of Agriculture, Vellayani campus ( $8^{\circ} 25^{\prime} 47.5^{\prime \prime} \mathrm{N}, 76^{\circ} 59^{\prime}$ 8.3" E) and Kallar Reserve Forest ( $8^{\circ} 41^{\prime} 48.9^{\prime \prime} \mathrm{N}, 7^{\circ} 7^{\prime} 53.6^{\prime \prime}$ E). All beetles were collected either from cashew or mango trees, except two specimens attracted to light.

Material from the following collections is examined:

$\mathrm{BMNH}=$ Natural History Museum, London, UK

$\mathrm{KAU}=$ Travancore Insect Collection, Kerala Agricultural University, Vellayani, India

MCZ = Museum of Comparative Zoology, Harvard University, Cambridge, MA, USA 


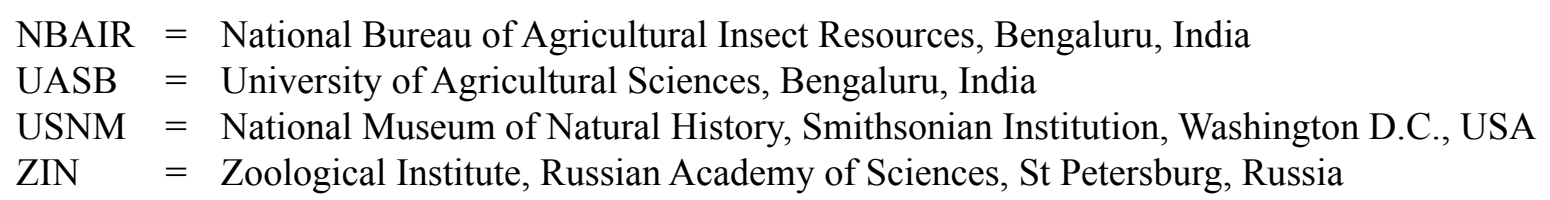

\section{Dissection}

The specimens were relaxed by immersing in soap solution for about 20 minutes. The abdomen was cut off from the body by inserting a no. 3 sized insect pin between the metasternum and the abdomen and then severing the membranous connections and tissues in between. The severed abdomen was macerated in $10 \% \mathrm{KOH}$ solution by keeping it overnight in a hot air oven, set at $50^{\circ} \mathrm{C}$. Alternatively, for quick processing, the specimens were boiled in $10 \% \mathrm{KOH}$ for about two minutes. The former method yielded better results as all the relevant structures, including ducts of the reproductive system, were intact, especially in females. The digested abdomen was thoroughly washed in water, followed by immersion in $2 \%$ acetic acid to remove excess alkali. One more round of washing with water was done to remove excess chemicals. The tergites and sternites were detached and the genitalia were completely pulled out of the muscles and tissues. The transparent membranous structures were stained with acid fuchsin and then transferred to glycerin for observation, photography and preservation.

\section{Genitalia}

The terminology for genitalia and associated structures follows Konstantinov (1998), Jolivet \&Verma (2008) and Moseyko (2008). The endophallus was everted through the apical opening (ostium) as well as inflated through basal opening. Inflation of the endophallus follows Yamasako \& Obhayashi (2011) with appropriate modification. A TSK STERiJECT hypodermic needle $0.2 \times 9 \mathrm{~mm}(3 / 8 ")$ (The invisible needles ${ }^{\mathrm{TM}}$ Japan) was mounted on a syringe filled with $\mathrm{K}-\mathrm{Y}^{\circledR}$ gel. The tip of the needle was inserted into the apical opening and affixed with the cyanoacrylate glue Fevikwik ${ }^{\circledR}$. After allowing five minutes to dry, the aedeagus was kept in water for a minute, then immersed in $\mathrm{K}-\mathrm{Y}^{\circledR}$ gel in a cavity slide and inflated by pushing the plunger flange slowly under controlled pressure. Eversion of endophallus was carried out using KK-3 type $\mathrm{C}$ fine nozzle. The endophallus was pushed towards the apical opening using the blunt end of a flexible fine needle through the basal opening. The tip of the fine nozzle, filled with $\mathrm{K}-\mathrm{Y}^{\circledR}$ gel, was affixed at the base of the aedeagus proper with cyanoacrylate glue (Fevikwik $\left.{ }^{\circledR}\right)$. The assembly was allowed to dry for five minutes and kept in water for one minute to relax the endophallic membrane. Then the aedeagus was immersed in K-Y ${ }^{\circledR}$ gel in a glass slide. The endophallus was then everted under controlled pressure of a syringe filled with $\mathrm{K}-\mathrm{Y}^{\circledR}$ gel.

Measurements were taken using an ocular micrometer. Images were stacked using Zerene stacker ver. 1.04. Photographs and stacked images were edited with Adobe Photoshop CS4. A line diagram of female genitalia was drawn using Adobe Illustrator.

\section{Biology}

Attempts were made to study the biology of F. kanaraensis. Live beetles were collected from the field and pairs of beetles were released into transparent plastic jars with a layer of top soil and a twig of mango or cashew with new growth. The mouths of the jars were covered with cloth and fastened with rubber bands. Beetles were allowed to mate and oviposit. The leaves were changed at three-day intervals, and the layer of soil was kept moist by spraying water. 
Table 1 (continued on the next page). Host plants of Fidia Motschulsky, 1861.

\begin{tabular}{|c|c|c|c|}
\hline \multicolumn{2}{|c|}{ No. Species } & $\begin{array}{l}\text { Host family and species } \\
\text { Carpinaceae: Carpinus L. } \\
\text { Fagaceae: Castanea Mill. } \\
\text { Quercus L. } \\
\text { Hamamelidaceae: Corylopsis Siebold \& Zucc. } \\
\text { Juglandaceae: Juglans L. }\end{array}$ & $\begin{array}{l}\text { Reference } \\
\text { Jolivet \& Hawkeswood } 1995\end{array}$ \\
\hline 1 & Fidia sp. & $\begin{array}{l}\text { Carpinaceae: Carpinus L. } \\
\text { Fagaceae: Castanea } \text { Mill. } \\
\text { Quercus L. } \\
\text { Hamamelidaceae: Corylopsis Siebold \& Zucc. } \\
\text { Juglandaceae: Juglans L. } \\
\quad \text { Pterocarya Nutt. ex Moq. } \\
\text { Lauraceae: Cinnamomum Schaeff. } \\
\quad \text { Machilus Desr. } \\
\text { Rosaceae: Malus Mill. } \\
\text { Prunus L. } \\
\text { Pyrus L. } \\
\text { Sapindaceae: Aesculus L } \\
\text { Styracaceae: Styrax L. } \\
\text { Taxodiaceae: Cryptomeria D.Don } \\
\text { Ulmaceae: Zelkova } \text { Spach. }\end{array}$ & Jolivet \& Hawkeswood 1995 \\
\hline 2 & $\begin{array}{l}\text { F. anger (Chûjô, 1935) } \\
\text { (= L. fulvus Baly, 1878) }\end{array}$ & Lauraceae: Cinnamomum daphnoides Sieb \& Zucc & Chûjô 1958a \\
\hline 3 & F. itoi (Chûjô, 1954) & Cupressaceae: Cryptomeria japonica D.Don & $\begin{array}{l}\text { Chûjô 1954; Ohno 1958; } \\
\text { Chûjô \& Kimoto 1961; } \\
\text { Kimoto } 1964\end{array}$ \\
\hline \multirow[t]{14}{*}{4} & F. atra (Motschulsky, 1861) & Betulaceae: Carpinus tschonoskii Maxim. & $\begin{array}{l}\text { Ohno 1958; } \\
\text { Chûjô \& Kimoto 1961; } \\
\text { Kimoto } 1964\end{array}$ \\
\hline & & $\begin{array}{l}\text { Fagaceae: Castanea crenata Sieb \& Zucc. } \\
\qquad \text { Quercus acutissima Carruthers }\end{array}$ & $\begin{array}{l}\text { Chûjô \& Kimoto 1961; } \\
\text { Kimoto 1964; Lee } 2012 \\
\text { Ohno 1958; } \\
\text { Chûjô \& Kimoto 1961; } \\
\text { Kimoto } 1964\end{array}$ \\
\hline & & Hamamelidaceae: Corylopsis spicata Sieb \& Zucc & $\begin{array}{l}\text { Chûjô 1954; Ohno 1958; } \\
\text { Chûjô \& Kimoto 1961; } \\
\text { Kimoto } 1964\end{array}$ \\
\hline & & Hippocastanaceae: Aesculus turbinata Blume & $\begin{array}{l}\text { Chûjô \& Kimoto 1961; } \\
\text { Kimoto } 1964\end{array}$ \\
\hline & & $\begin{array}{l}\text { Juglandaceae: Juglans mandshurica Maxim. } \\
\text { Juglans ailanthifolia Carruthers }\end{array}$ & $\begin{array}{l}\text { Chûjô 1954; Ohno } 1958 \\
\text { Chûjô \& Kimoto 1961; } \\
\text { Kimoto } 1964\end{array}$ \\
\hline & & Juglans sinensis Dode & Lee 2012 \\
\hline & & Pterocarya rhoifolia Sieb \& Zucc & Ohno 1958 \\
\hline & & Rosaceae: Malus pumila (Willd.) & $\begin{array}{l}\text { Chûjô 1954; Ohno 1958; } \\
\text { Chûjô \& Kimoto 1961; } \\
\text { Kimoto 1964; Lee 2012 }\end{array}$ \\
\hline & & Malus prunifolia (Willd) & $\begin{array}{l}\text { Chûjô \& Kimoto 1961; } \\
\text { Kimoto } 1964\end{array}$ \\
\hline & & Pyrus pyrifolia (Burm.f.) & Lee 2012 \\
\hline & & Pyrus asiatica (Nakai) M.F.Fay \& Christenh. & Chen 1940; Lee 2012 \\
\hline & & Prunus muтe Sieb \& Zucc & $\begin{array}{l}\text { Ohno 1958; } \\
\text { Chûjô \& Kimoto 1961; } \\
\text { Kimoto 1964; Lee } 2012\end{array}$ \\
\hline & & Styracaceae: Styrax japonica Sieb \& Zucc & $\begin{array}{l}\text { Ohno 1958; } \\
\text { Chûjô \& Kimoto 1961; } \\
\text { Kimoto } 1964\end{array}$ \\
\hline & & Ulmaceae: Zelkova serrata (Thumb.) & $\begin{array}{l}\text { Ohno 1958; } \\
\text { Chûjô \& Kimoto 1961; } \\
\text { Kimoto } 1964\end{array}$ \\
\hline
\end{tabular}


Table 1 (continued). Host plants of Fidia Motschulsky, 1861.

\begin{tabular}{|c|c|c|c|}
\hline No. & Species & Host family and species & Reference \\
\hline 5 & F. fulva (Baly, 1878) & $\begin{array}{l}\text { Lauraceae: Cinnamomum daphnoides Sieb \& Zucc } \\
\qquad \text { Machilus thunbergii Sieb.\& Zucc } \\
\text { Fagaceae: Quercus salicina Blume }\end{array}$ & $\begin{array}{l}\text { Chûjô \& Kimoto 1961; } \\
\text { Kimoto \& Gressitt } 1966 \\
\text { Kimoto \& Gressitt } 1966 \\
\text { Chûjô \& Kimoto 1961; } \\
\text { Kimoto \& Gressitt } 1966\end{array}$ \\
\hline 6 & F. kanaraensis (Jacoby, 1895) & $\begin{array}{c}\text { Anacardiaceae: Anacardium occidentale } \mathrm{L} \text {. } \\
\text { Mangifera indica } \mathrm{L} .\end{array}$ & $\begin{array}{l}\text { New record } \\
\text { Rajendran \& Singh } 2016\end{array}$ \\
\hline 7 & F. kiiensis (Ohno, 1958) & Fagaceae: Quercus L. & $\begin{array}{l}\text { Ohno 1958; } \\
\text { Chûjô \& Kimoto 1961; } \\
\text { Kimoto } 1964\end{array}$ \\
\hline 8 & F. lewisii (Baly, 1878) & Lauraceae: Machilus thunbergii Sieb \& Zucc & $\begin{array}{l}\text { Ohno 1958; } \\
\text { Chûjô \& Kimoto 1961; } \\
\text { Kimoto } 1964\end{array}$ \\
\hline
\end{tabular}

\section{Results}

Genus Neofidia Strother nom. nov.

Fidia Dejean, 1836: 412 (catalog), nom. nud.

Atonia Gistel, 1848: 123 (nec Williston 1889, Diptera: Asilidae) (synonym of Fidia Dejean), nom. nud. Fidia Baly, 1863: 153 (nec Motschulsky 1861: 22) (original description).

Fidea Glover, 1868: 71 (misspelling).

Fidia Dejean, 1836 - Dejean 1837: 436 (catalog). - Sturm 1843: 295 (catalog). — Crotch 1873: 33 (faunal treatment, species description). — Chapuis 1874: 275 (classification).

Atonia - Gistel 1851: 123.

Fidia Baly, 1863 - Gemminger \& Harold 1874: 3376 (catalog). — Lefèvre 1877: 164 (species descriptions); 1885: 75 (catalog, species description). - Henshaw 1885: 108 (catalog). — Lefèvre 1885: 75 (catalog, species description). - Horn 1892: 198 (faunal treatment, species description). — Blatchley 1910: 1142 (faunal treatment). — Clavareau 1914: 76 (catalog). — Leng 1920: 293 (catalog). - Blackwelder 1946: 662 (checklist). - Bechyné 1950: 288 (taxonomic treatment); 1953: 249 (catalog). - Wilcox 1954: 402 (faunal treatment). — Arnett 1963: 928 (faunal treatment). — Balsbaugh \& Hays 1972: 73 (faunal treatment). — Riley et al. 2003: 151 (catalog). — Ciegler 2007: 168 (faunal treatment). — Strother et al. 2007: 36 (nomenclature).

Fidia - Walsh, 1867: 87 (species description). — Wilcox 1975: 57 (checklist). — Seeno \& Wilcox 1982: 62 (checklist). — Arnett 1985: 376 (faunal treatment). — LeSage 1991: 309 (faunal treatment). — Flowers 1996: 36 (checklist). — Riley et al. 2002: 671 (catalog).

\section{Type species}

Fidia lurida Baly, 1863: 153 (not Fidia lurida Dejean, 1836, nom. nud.)

\section{Etymology}

The name Neofidia alludes to the new name for Fidia Baly, 1863, as well as its Nearctic and Neotropical distribution. The gender is feminine. 


\section{Checklist of species}

Neofidia albovittata (Lefèvre, 1877) comb. nov.

Fidia albovittata Lefèvre, 1877: 166

Fidia sallei Lefèvre, 1877: 166

Fidia sallaei Jacoby, 1882: 167 [unjustified emendation]

Fidia unistriata Jacoby, 1882: 168

Neofidia cana (Horn, 1892) comb. nov.

Fidia cana Horn, 1892: 199 (Fidia)

Neofidia clematis (Schaeffer, 1904) comb. nov.

Fidia clematis Schaeffer, 1904: 227

Neofidia comalensis (Strother in Strother \& Staines, 2008) comb. nov.

Fidia comalensis Strother in Strother \& Staines, 2008: 29

Neofidia confusa (Strother, 2003) comb. nov.

Fidia confusa Strother, 2003: 151

Fidia murina Crotch, 1873: 33 [not Glover, 1868: 71]

Neofidia convexicollis (Strother in Strother \& Staines, 2008) comb. nov.

Fidia convexicollis Strother in Strother \& Staines, 2008: 33

Neofidia costaricensis (Strother in Strother \& Staines, 2008) comb. nov.

Fidia costaricensis Strother in Strother \& Staines, 2008: 35

Neofidia delilahae (Strother in Strother \& Staines, 2008) comb. nov.

Fidia delilahae Strother in Strother \& Staines, 2008: 37

Neofidia dicelloposthe (Strother in Strother \& Staines, 2008) comb. nov.

Fidia dicelloposthe Strother in Strother \& Staines, 2008: 38

Neofidia dichroma (Strother in Strother \& Staines, 2008) comb. nov.

Fidia dichroma Strother in Strother \& Staines, 2008: 39

Neofidia guatemalensis (Jacoby, 1879) comb. nov.

Fidia guatemalensis Jacoby, 1879: 778

Neofidia humeralis (Lefèvre, 1877) comb. nov.

Fidia humeralis Lefèvre, 1877: 165

Fidia plagiata Lefèvre, 1877: 165

Neofidia longipes (Melsheimer, 1847) comb. nov.

Eumolpus longipes Melsheimer, 1847: 169

Pachnephorus viticolus Uhler, 1855: 418

Neofidia lurida (Baly, 1863) comb. nov.

Fidia lurida Baly, 1863: 153

Colaspis flavescens Sturm, 1826: 123 [nom. nud.]

Fidia lurida Dejean, 1836: 412 [nom. nud.]

Fidia viticida Walsh, 1867: 87

Neofidia marraverpa (Strother in Strother \& Staines, 2008) comb. nov.

Fidia marraverpa Strother in Strother \& Staines, 2008: 49

Neofidia papillata (Strother in Strother \& Staines, 2008) comb. nov.

Fidia papillata Strother in Strother \& Staines, 2008: 50

Neofidia pedestris (Lefèvre, 1877) comb. nov.

Fidia pedestris Lefèvre, 1877: 164

Neofidia pedinops (Strother in Strother \& Staines, 2008) comb. nov.

Fidia pedinops Strother in Strother \& Staines, 2008: 54

Fidia murina Dejean, 1836: 412 [nom. nud.]

Neofidia rileyorum (Strother in Strother \& Staines, 2008) comb. nov.

Fidia rileyorum Strother in Strother \& Staines, 2008: 56 
Neofidia spuria (Lefèvre, 1877) comb. nov.

Fidia spuria Lefèvre, 1877: 165

Fidia atra Jacoby, 1882: 168 (not Motschulsky, 1861)

Neofidia texana (Schaeffer, 1933) comb. nov.

Fidia viticida var. texana Schaeffer, 1933: 472

Neofidia tibialis (Jacoby, 1890) comb. nov.

Fidia tibialis Jacoby, 1890: 232

Neofidia xanthonioides (Strother in Strother \& Staines, 2008) comb. nov.

Fidia xanthonioides Strother in Strother \& Staines, 2008: 94

\section{Remarks}

Strother \& Staines (2008) provided a diagnosis and description of the genus. Both the genera, Fidia Motschulsky, 1861 and Neofidia Strother nom. nov., belong to the same tribe, Adoxini Baly, 1863 (sensu Moseyko \& Kirejtshuk 2013). The Old World genus Fidia can be separated from the New World genus Neofidia Strother nom. nov. by the presence of a tooth on the ventral side of all femora (absent in Neofidia Strother nom. nov.). Another character mentioned by Strother \& Staines (2008), the absence of paired apical spurs on the apex of fore tibia, cannot be used for separating these genera as spurs are present in some species of Fidia, at times being small and poorly visible. Among the Old World genera, Neofidia Strother nom. nov. is mostly close to Aoria Baly, 1863 and the similar genera Aloria Bryant, 1939 and Bromius Chevrolat in Dejean, 1836. It can be separated from Aoria by simple intercoxal mesothoracic process (bifurcated or with two spines in Aoria), from Aloria by 11-segmented antennae (9-segmented in Aloria) and from Bromius by the straight fore margin of lateral arms of pronotum ("propleura" of Jacoby 1908; convex in Bromius).

\section{Distribution}

North and Central America (Strother \& Staines 2008)

\section{Generic diagnosis}

Body oblong, punctate-setose, frons and vertex hardly differentiated, antenna filiform, longer than $1 / 2$ to $3 / 4$ of body length, second antennomere shorter than first, longer than half of it, shorter than next four or five. Compound eyes lateral, convex and entire, antennal calli triangular, pronotum as long as wide or gently wider than long, narrower than elytra, without lateral margins, fore margin of lateral arms of pronotum ('propleuron') straight, legs long and slender, tibia longitudinally carinate, all femora lack a ventral tooth, intermediate and posterior tibiae not emarginate preapically, apex of each tibia with a pair of small spurs, claws bifid.

Genus Fidia Motschulsky, 1861

Fidia Motschulsky, 1861: 22 (Strother et al. 2007 referred to International Commission on Zoological Nomenclature (ICZN) for suppression); ICZN (2009) rejected Strother et al. 2007 and reaffirmed priority.

Endoxus Baly, 1861: 285 (misspelling).

Lypesthes Baly, 1863: 152, type species Fidia atra Motschulsky, 1861.

Leprotes Baly, 1863: 158, type species Adoxus gracilicornis Baly, 1861 (synonymized with Lypesthes Baly, 1863).

Talmonus Fairmaire, 1889: 71, type species Talmonus farinosus Fairmaire, 1889 (Fidia atra Motschulsky, 1861) (synonymised with Lypesthes Baly, 1863).

Lypesthinia Pic, 1939: 32 (valid subgenus) [type species Lypesthinia multidentata Pic, 1939: 32]. 
Lypesthes - Chapuis 1874: 273. — Gemminger \& Harold 1874: 3376 (catalog). — Lefèvre 1885: 74. - Jacoby 1908: 395, 412. - Clavareau 1914: 76 (catalog). — Chen 1935: 370-371; 1940: 491. —Chûjô 1938: 30; 1956: 88, 101. — Ohno 1958: 178. — Gressitt \& Kimoto 1961: 198, 269. — Chûjô \& Kimoto 1961: 145. — Kimoto 1964: 256; 2005: 34. — Kimoto \& Gressitt 1982: 73. — Seeno \& Wilcox 1982: 62 (checklist). — Moseyko \& Sprecher-Uebersax 2010: 624.

Leprotes - Baly 1878: 250. — Chapuis 1874: 273. —Lefèvre 1885: 80. — Jacoby 1885: 203; 1908: 396, 425-426. - Chen 1935: 370-371.

Talmonus - Jacoby 1890: 115.

Lypesthinia - Seeno \& Wilcox 1982: 62 (checklist).

\section{Type species}

Fidia atra Motschulsky, 1861: 22 (not Fidia atra Jacoby, 1882).

\section{Checklist of species}

Fidia albida (Pic, 1923) comb. nov. Lypesthes albidus Pic, 1923: 16

Fidia atra Motschulsky, 1861: 22

Leprotes pulverulentus Jacoby, 1885: 203

Talmonus farinosus Fairmaire, 1889: 71

Leprotes testaceipes Pic, 1928: 26

Lypesthes ater fulvipes Chûjô, 1954: 106

Lypesthes ater ater f. tibialis Ohno, 1958: 174

Fidia basalis (Chen, 1940) comb. nov.

Leprotes atra Pic, 1936: 15

Leprotes basalis Chen, 1940: 517 [replacement name for Leprotes atra Pic, 1936: 15]

Fidia bicoloripes (Pic, 1936) comb. nov.

Leprotes bicoloripes Pic, 1936: 15

Fidia bisquamosa (Chen, 1935) comb. nov. Lypesthes bisquamosus Chen, 1935: 374

Fidia brancuccii (Medvedev, 1993) comb. nov. Hyperaxis brancuccii Medvedev, 1993: 362

Fidia carinata (Eroshkina, 1992) comb. nov. Lypesthes carinatus Eroshkina, 1992: 86

Fidia fulva (Baly, 1878) comb. nov. Leprotes fulvus Baly, 1878: 250

Lypesthes anger Chûjô, 1935: 204

Lypesthes taiwanus Chûjô, 1956: 105

Fidia gracilicornis (Baly, 1861) comb. nov. Adoxus gracilicornis Baly, 1861: 285

Lypesthes sauteri Chûjô, 1938: 30

Fidia impressa (Pic, 1928) comb. nov.

Leprotes impressus Pic, 1928: 26

Fidia indica (Jacoby, 1908) comb. nov. Lypesthes indica Jacoby, 1908: 412

Fidia irregularis (Eroshkina, 1992) comb. nov. Lypesthes irregularis Eroshkina, 1992: 88

Fidia itoi (Chûjô, 1954) comb. nov. Lypesthes itoi Chûjô, 1954: 103

Fidia japonica (Ohno, 1958) comb. nov. 
Lypesthes japonicus Ohno, 1958: 179

Lypesthes babai Chûjô, 1958b: 4

Fidia kanaraensis (Jacoby, 1895) comb. nov.

Leprotes kanaraensis Jacoby, 1895: 270

Fidia kiiensis (Ohno, 1958) comb. nov.

Lypesthes kiiensis kiiensis Ohno, 1958: 177

Lypesthes kiiensis ab immaculatus Ohno, 1958: 179

Fidia laeta (Medvedev, 2007) comb. nov.

Lypesthes laetus Medvedev, 2007: 8

Fidia lewisi (Baly, 1878) comb. nov.

Leprotes lewisi Baly, 1878: 251

Fidia mausonica (Eroshkina, 1992) comb. nov.

Lypesthes mausonica Eroshkina, 1992: 87

Fidia medvedevi nom. nov.

Lypesthes vietnamicus Medvedev, 2015: 324 (nec Lypesthes vietnamicus Eroshkina, 1992: 89)

Fidia multidentata (Pic, 1939) comb. nov.

Lypesthinia multidentata Pic, 1939: 32

Fidia perelegans (Gressitt \& Kimoto, 1961) comb. nov.

Lypesthes perelegans Gressitt \& Kimoto, 1961: 271

Fidia phoebicola (Tan, 1983) comb. nov.

Hyperaxis phoebicola Tan, 1983: 130

Fidia picea (Gressitt \& Kimoto, 1961) comb. nov.

Lypesthes piceus Gressitt \& Kimoto, 1961: 271

Fidia regalis (Medvedev \& Zoia, 1996) comb. nov.

Lypesthes regalis Medvedev \& Zoia, 1996: 116

Fidia rufa (Pic, 1924) comb. nov.

Leprotes rufus Pic, 1924: 6

Fidia shirozui (Kimoto, 1969) comb. nov.

Lypesthes shirozui Kimoto, 1969: 17

Lypesthes farinosus Chûjô, 1938: 30 (nec Pic, 1923, nec Fairmaire, 1889)

Lypesthes gracilicornis Chûjô, 1956: 106 (nec Baly, 1861)

Fidia sinensis (Gressitt \& Kimoto, 1961) comb. nov.

Lypesthes sinensis Gressitt \& Kimoto, 1961: 272

Fidia striata (Eroshkina, 1992) comb. nov.

Lypesthes striatus Eroshkina, 1992: 84

Fidia submaculata (Pic, 1924) comb. nov.

Leprotes submaculatus Pic, 1924: 6

Fidia subregularis (Pic, 1923) comb. nov.

Leprotes subregularis Pic, 1923: 17

Leprotes farinosus Pic, 1923: 17

Fidia sulcatifrons (Gressitt \& Kimoto, 1961) comb. nov. Lypesthes sulcatifrons Gressitt \& Kimoto, 1961: 273

Fidia vietnamica (Eroshkina, 1992) comb. nov.

Lypesthes vietnamicus Eroshkina, 1992: 89

Fidia vittata (Zhou \& Tan, 1997) comb. nov.

Lypesthes vittatus Zhou \& Tan, 1997: 189

\section{Remarks}

For comparison of Fidia with the genus Neofidia Strother nom. nov., see 'Remarks' under Neofidia Strother nom. nov. The Old World genus Fidia belongs to a comparatively large genus-group of the 
tribe Adoxini, including the genera with bifid claws, straight fore margin of lateral arms of pronotum ('propleura'), simple intercoxal mesothoracic process and tooth on underside of each femur. It is poorly delimited from the genus Hyperaxis Gemminger \& Harold, 1874, having a difference in longer pronotum, narrower hind femora with smaller tooth and smaller eyes.

\section{Distribution}

China, Indo-China, India, Japan, Korea, Laos, Myanmar, Nepal, Pakistan, Taiwan, Thailand, Vietnam.

\section{Generic diagnosis}

Body oblong, adorned with setae and or scales. Frons merges with vertex, hardly differentiated. Antenna filiform, longer than half of body length, longer than body length in some cases. Second antennomere usually shorter than first, longer than half of it, shorter than next three or four. Compound eyes lateral, hemispherical, feebly or not emarginate near antennal sockets. Antennal calli absent. Pronotum longer than wide or subquadrate, narrower than elytra, lateral margins incomplete, fore margin of lateral arms of pronotum ('propleuron') straight. Pygidium with or without elytral locking groove. Legs long, slender, all femora slightly thickened and toothed beneath, all tibiae longitudinally sulcate with rows of setae, mid- and hind tibiae not emarginate at apex, with short stout paired spines on apex. Claws bifid.

Fidia kanaraensis (Jacoby, 1895) comb. nov.

Figs 1-22, 24-25

Leprotes kanaraensis Jacoby, 1895: 270.

Leprotes kanaraensis - Jacoby 1908: 426. — Clavareau 1914: 76 (catalog).

\section{Material examined}

Type material

INDIA 11 spec. (syntype of Leprotes kanaraensis, right antenna without five distal antennomeres; left antenna without three distal antennomeres; tibia and tarsi of right foreleg missing); Kanara; Jacoby leg; NHM 1909-28a (Fig. 18) • 1 spec. (syntype of Leprotes kanaraensis, labelled as "Cotype"); Kanara; H.E. Andrewes Bequest leg.; BMNH B.M. 1922-221 (Fig. 19) • 1 spec. (syntype of Leprotes kanaraensis, labelled as "Cotype"); Mahe, Malabar; MCZ Type 9764 (images available from: http://140.247.96.247/mcz/Species_record.php?id=9383).

Other material (44 specimens)

INDIA • 1 unsexed; India, Kerala, Vellayani; $08^{\circ} 25^{\prime} 47.5^{\prime \prime}$ N, $76^{\circ}$ 59'8.3" E; 18 m a.s.1.; 12 May 2017; Amritha Kumari leg.; cashew; KAU • 1 क; same collection data as for preceding; 12 Apr. 2017; KAU • 1 unsexed; same collection data as for preceding; 13 Jun. 2017; KAU $\bullet 3$ unsexed; same collection data as for preceding; 15 Jun. 2017; KAU • 1 unsexed; same collection data as for preceding; 26 Jun. 2017; KAU 1 unsexed; same collection data as for preceding; 24 Jun. 2017; H. Sangamesh leg.; at light; KAU - 1 unsexed; same collection data as for preceding; 15 May 2019; KAU • 1 ภ, 1 + , 1 unsexed; Kerala,

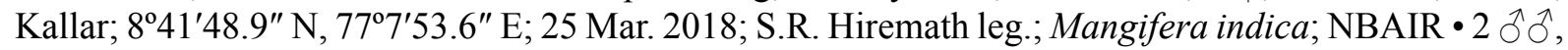

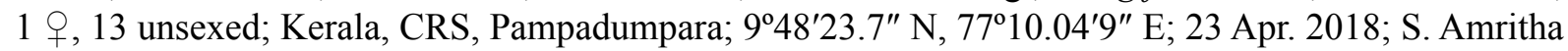
Kumari. leg.; mango; NBAIR 11 unsexed; Karnataka, Bangalore; 916 m a.s.1.; 27 May 2006; K.J. David leg.; mango; UASB • 2 unsexed; Karnataka, Bangalore, GKVK; 8 Jun. 2006; K.S. Girish leg.; Mangifera indica; UASB - 1 unsexed; Karnataka, Bangalore, Hessarghatta; 12 Jul. 2009; H.M. Yeshwanth; UASB - 1 unsexed; Karnataka, Bangalore, Attur, 10 May 2012; mango; C.A. Viraktamath leg.; UASB • 1 unsexed; S Malabar, Walayar; 2000 ft. a.s.l.; 8 Aug. 1938; UASB • 1 unsexed; N Malabar, Taliparamba; 4 Aug. 1927; A.G.R. Coll leg.; pepper; UASB • 3 unsexed; Karnataka, Shanthigodu; Apr. 1988; cashew 
sp. 17, CIE A20806, Pres. by Comm. Inst. Entomol. BM1990-1; identified by M.L. Cox as Neculla ?pollinaria; BMNH - 1 unsexed; Sindhudurg, Vengurla; 16 Apr. 1989; ?mango/cashew sp. 60, N. Khochare, CIE A20874, Pres. by Comm. Inst. Entomol. BM1990-1; identified by M.L. Cox as Neculla ?pollinaria; BMNH • 1 unsexed; Côte de Malabar; T. Deschamps leg.; 1900; F. Monros leg.; USNM • 2 unsexed; Chota-Nagpore, Barway, P. Cardon; Jun.-Aug. 1896; F. Monros leg.; USNM • 2 unsexed; Kerala, Chalakudy; 9 May 2008; J. Thomas leg.; cashew; ZIN • 1 unsexed; Kerala, Erattupettah, Wagamon Pass; 25 Apr. 2010; Prathapan leg.; ZIN.

\section{Redescription}

Body Length. 4.6-5.0 mm (male); 6.4-7.2 mm (female). Width 2.6-2.8 mm (male); 3.3-3.9 mm (female). Body covered with white powdery encrustation (Figs 2-4, 25) absent on antennae, eyes, mouth parts and distal portion of legs. Integument entirely black except labrum, labium, maxilla, basal three or four antennomeres and claws dark chocolate brown. Entire dorsum covered with creamy white, dorsoventrally flattened, erect or curved pointed setae (Figs 1, 4).

HEAD. As densely setose as pronotum; punctures dense and coarse, distance between adjacent punctures less than half of diameter of a puncture (Fig. 5). Frons flat or gently depressed medially; anteriorly sparsely setose, sloping with smaller punctures, merges with narrow clypeus; anterolateral corners obtusely projecting. Antenna exceeds half of body length in male, shorter in female. First antennomere curved, thick, proximally black, distally brown; second antennomere thinner than first; 3-6 thin; 7-11 progressively broadened. Ratio of length of antennomeres $1-11$ equals $1: 0.64-0.9: 0.7-0.8: 0.7-0.87$ : $0.87-0.93: 0.78-0.87: 1.04-1.16: 0.87-0.96: 0.9-1.01: 0.87-0.99: 1.16-1.19$ (male); $1: 0.68-0.78$ : $0.78: 0.85: 0.9: 0.78-0.83: 0.93-1.1: 0.78-0.85: 0.73-0.88: 0.75-0.85: 1-1.15$ (female). $8^{\text {th }}$ and $9^{\text {th }}$ antennomeres $1.8-2$ times longer than broad. Transverse diameter of eye subequal to vertical, 3.7 to 4.8 times distance between antennal socket and eye. Interoccular space 1.75 to 2 times wider than interantennal space in males, 1.3-1.5 times wider in females. Transverse diameter of eye about 2.9 times diameter of antennal socket in male, 3.4-3.6 times in female. Interantennal space 1.3-1.8 times transverse diameter of one eye. Maximum width of head 1.5-1.7 times interocular space, 2.6-2.9 times interantennal space. Clypeus indistinct, narrow, with very short setae, anterior margin concave. Labrum broad apically; anterior margin thick, sloping, feebly emarginate; with a transverse row of four setose punctures, anterolateral corners each with a group of three longer setae, anterior margin of labrum with a row of short setae on either side, absent in middle. Maxillary palpi with apical palpomere longest, broadest; penultimate palpomere apparently shorter than one preceding it. Labial palpi with apical palpomere longer than preceding one. Mandibles stout, with long, bent setae laterally.

Pronotum. 1.3-1.5 times broader than long; posteriorly 1.2-1.4 times wider than anteriorly; lateral margin weakly formed in posterior half, absent anteriorly, weakly but regularly convex. Anterolateral callosity rounded with setae bearing pore placed laterally, not distinctly protruding laterally; posterolateral callosity rounded with seta bearing pore slightly protruding laterally. Punctures on pronotum subequal to those on vertex, distinctly smaller than elytral ones.

ScUTELLUM. Longer than broad, apex weakly angulate presenting pentagonal appearance, with dense setae and punctures anteriorly, impunctate and shiny posteriorly.

ELYTRA. 1.28-1.38 times longer than broad. Elytra convex, sloping from apical third; lateral margins distinctly curved in middle; apex concave, obtusely angulate at sutural angle; humeral calli well developed with depression mesally and posteriorly; basal calli weakly but distinctly developed with weak depression posteriorly; punctures semiregular with a few countable rows in posterior half near sutural margin; interstices setose; setae flat, pointed. 


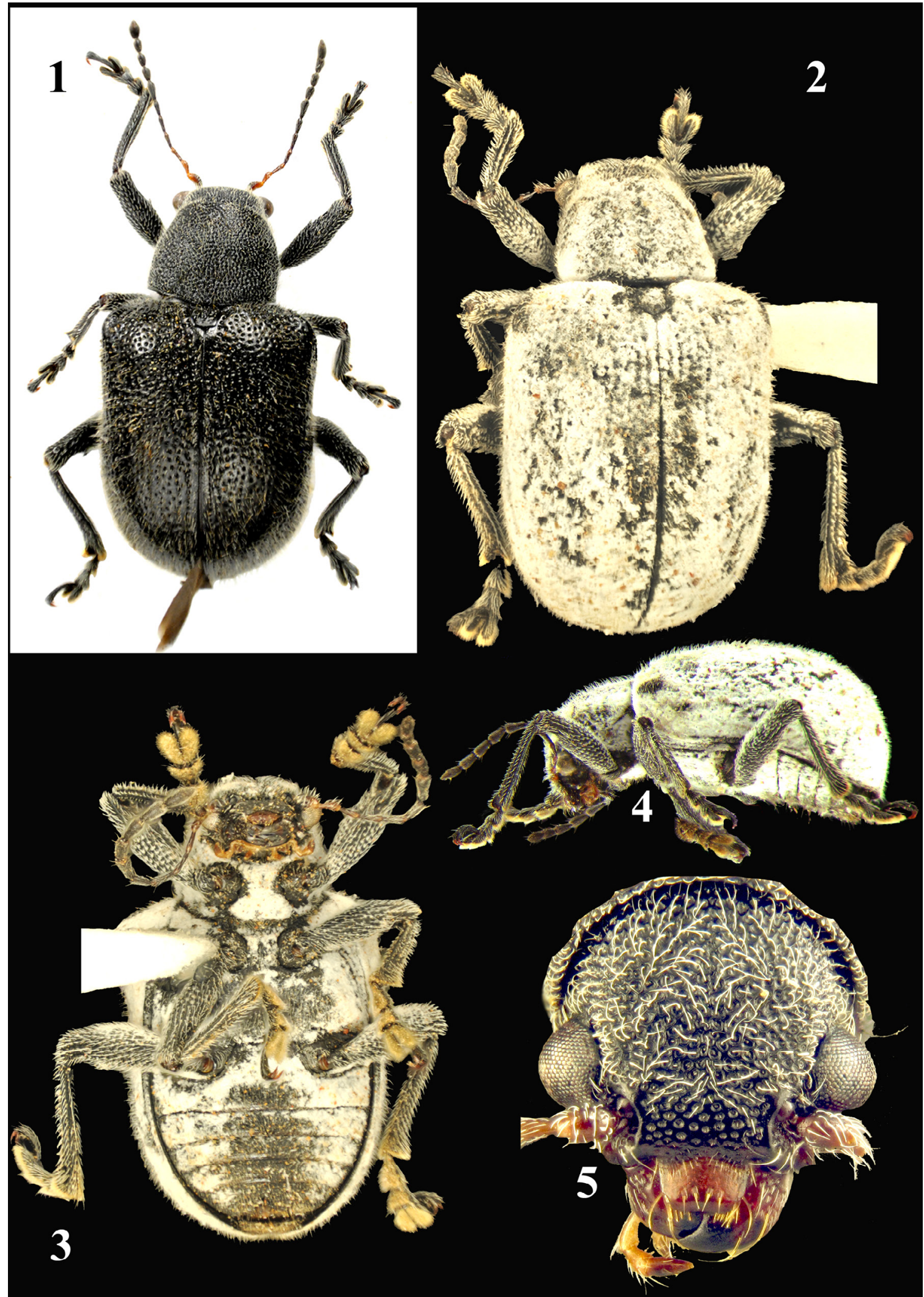

Figs 1-5. Fidia kanaraensis (Jacoby, 1895) comb. nov. 1. Dorsal view of habitus after removing powdery encrustation. 2. Dorsal view of habitus with powdery encrustation. 3. Ventral view of habitus. 4. Lateral view of habitus. 5. Head. 

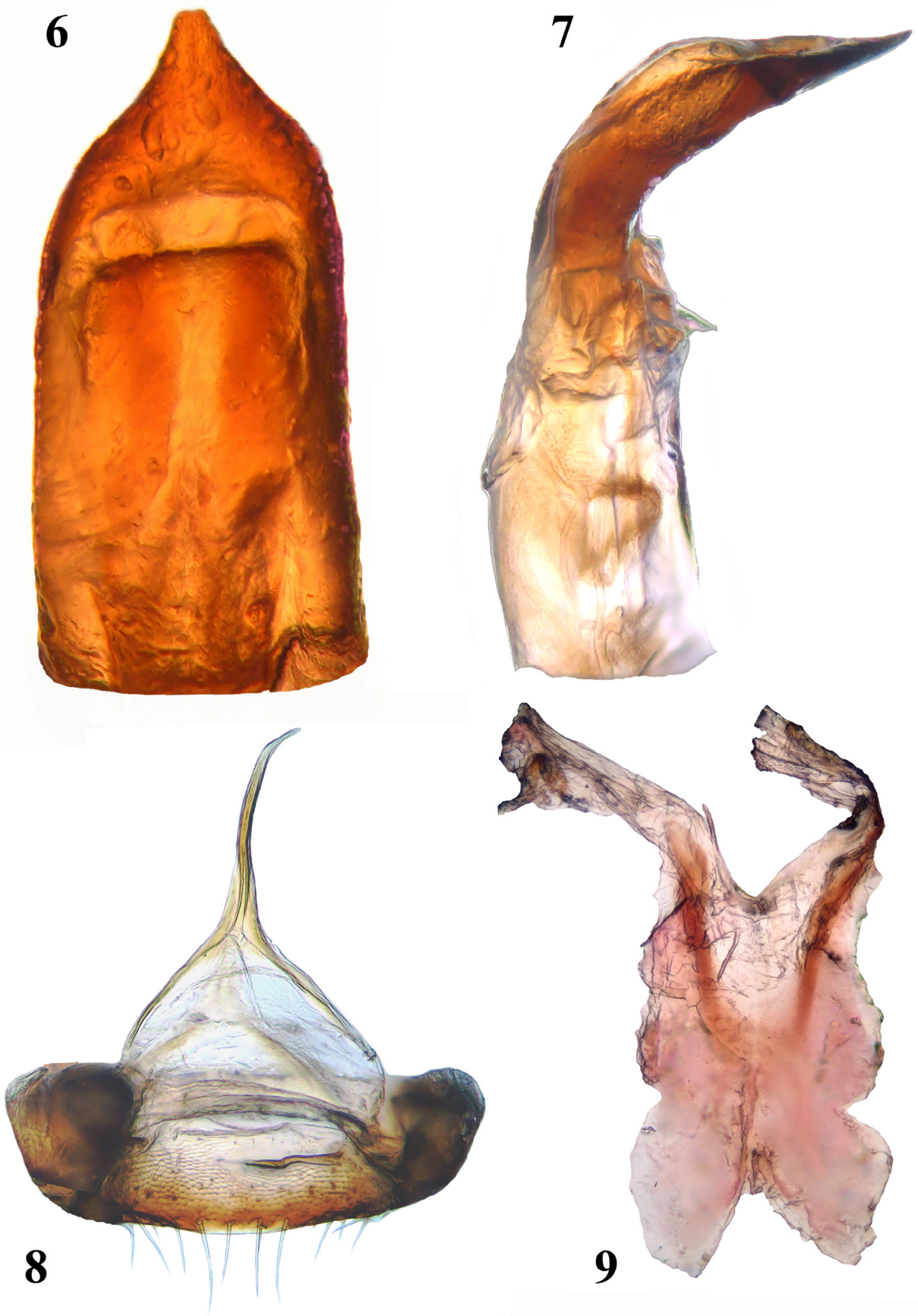

Figs 6-9. Male genitalia of Fidia kanaraensis (Jacoby, 1895) comb. nov. 6. Aedeagus, dorsal view. 7. Aedeagus, lateral view. 8. Tergite VIII, sternite VIII \& IX. 9. Tegmen. 
Thorax. Propleura with setose punctures. Thoracic sternites pubescent. Prosternum subquadrate with concave lateral margins, broadened posteriorly, densely punctate.

ABDomen. Ventrites setose; first ventrite longer than following two combined; first ventrite longest, ventrites 2-4 each shorter than preceding one, fifth longer than fourth, shorter than 3 and 4 combined.

Male Genitalia. Tegmen (first spiculum of Jolivet \& Verma 2008) membranous with ' $Y$ '-shaped sclerotization, bifurcated distally, basal region narrow, encircles aedeagus (Fig. 9). Aedeagus in ventral view broadest proximally with lateral margin slightly concave medially, narrowed towards apex; apex triangular with tip truncated, ventral surface concave medially, more or less flat distally (Fig. 6). Spiculum gastrale (second spiculum of Jolivet \& Verma 2008) Y-shaped with curved lateral arms, stem distinctly bent at tip. Tergite VIII semi-elliptical in shape with setose apical margin; spiculum relictum indistinct, Y-shaped (Fig. 8). Endophallus everted completely through apical opening, much longer than aedeagus proper with three distinct regions: basal phallomere (BP), median phallomere (MP) and apical phallomere (AP) (Fig. 10). Basal phallomere slightly shorter than median phallomere, narrowed in proximal half with a pair each of longitudinal sclerites dorsally and ventrally; distal half bulged with a large ventral bulb, covered with circular and triangular spicules. Median phallomere longest with a shorter proximal part and a longer distal part; proximal part with a small ventral bulb, membrane with a few circular spicules; distal part widest medially, membrane covered with small triangular spicules arranged serially. Apical phallomere globular, with three pairs of ventro-apical lobes (L1, L2 \& L3) and a bifurcated apical sclerite (AS). Endophallus, when inflated through basal opening (Figs 11-12), only part of median phallomere and apical phallomere were visible; basal phallomere completely and proximal region of median phallomere being hidden.

Female Genitalia (Fig. 13). With spermathecal capsule sickle shaped, weakly constricted proximally, apex subobtuse, inner margin almost V-shaped, outer margin strongly curved (Fig. 16). Length of spermathecal capsule 2.6-3.1 times its maximum width. Bursa copulatrix distinctly sclerotized, almost bean shaped (Fig. 14); spermathecal duct short, enters bursa copulatrix at its apex; spermathecal gland long, tubular, translucent, bifurcated in distal $2 / 3$, arise close to spermathecal duct. Tergite VIII simple and crescent-shaped (Fig. 17), bears a number of long setae on its apical margin. Sternite VIII unrecognizable and tignum sensu Konstantinov 1998 (spiculum ventrale sensu Ślipiński \& Escalona 2013) absent. Ovipositor very short, with partially reduced structures (Fig. 15). Proctiger is represented by a pair of baculi, connected basally with paraprocts. Paraprocts are small, connected with baculi of proctiger and valvifers. Valvifers are slightly elongate, coxites are with pointed apex and lack long setae on the apex. Styli are absent.

\section{Distribution}

India (Bengal, 'southern Bombay', Karnataka, Kerala).

\section{Host plants}

Anacardium occidentale L. (new record) and Mangifera indica L. (Rajendran \& Singh 2016) (both Anacardiaceae).

\section{Biology}

Fidia kanaraensis is a pest of cashew (Anacardium occidentale) and mango (Mangifera indica) in south India. Adults create moderate sized circular or irregular holes on the tender leaves (Fig. 26), mostly during the night and late evening hours, and hardly come out during the day. Infestation is apparently higher in cooler areas. Rajendran \& Singh (2016) listed it as a pest of mango. No further information was available on its immature stages or biology. This is the first record of F. kanaraensis on cashew. Eggs were laid singly on the surface of soil and covered with a mixture of excreta and soil (Fig. 20). 


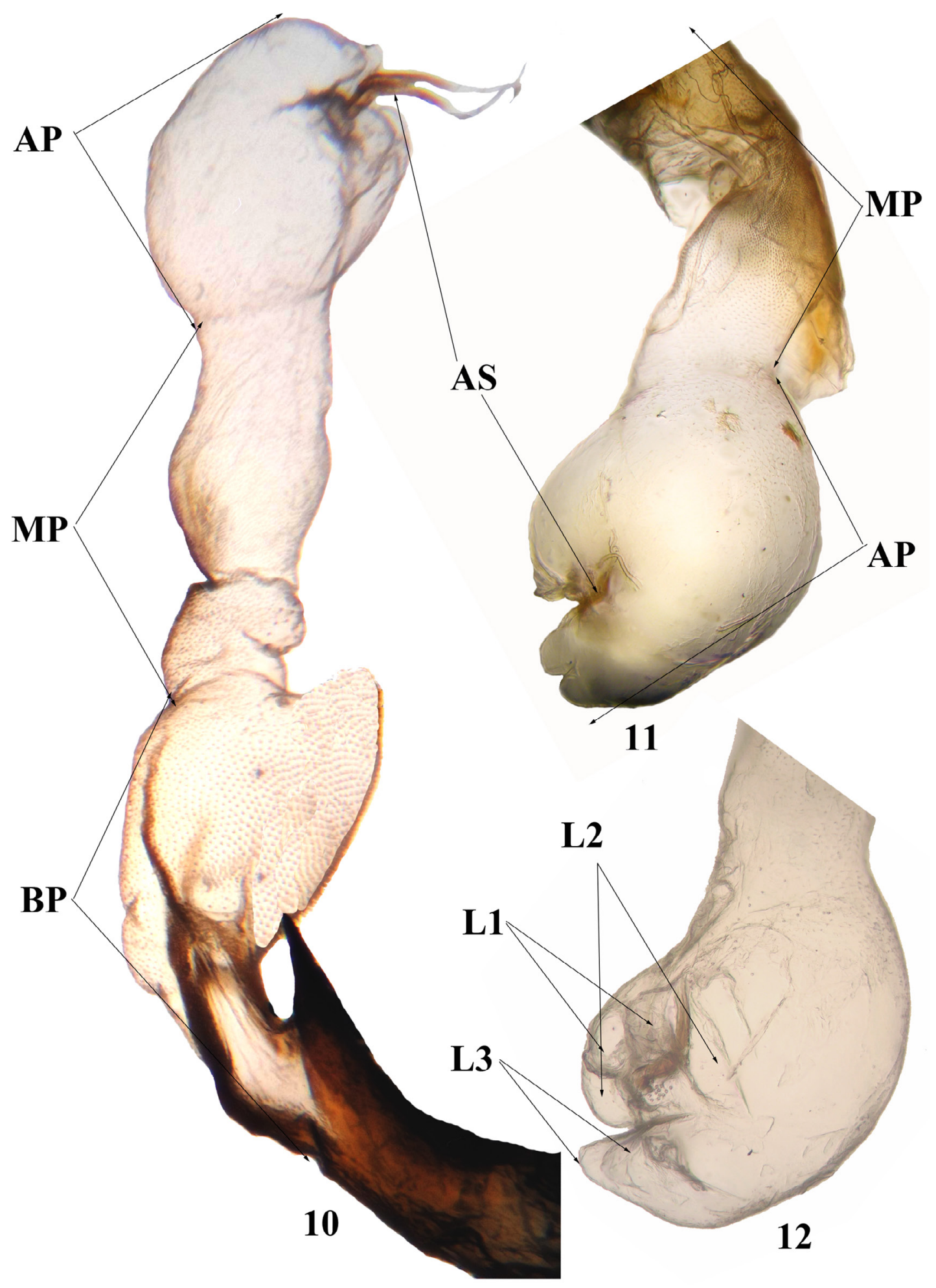

Figs 10-12. Endophallus of Fidia kanaraensis (Jacoby, 1895) comb. nov., lateral view. 10. Endophallus, everted through apical opening or ostium. 11. Endophallus, everted through basal opening. 12. Apical phallomere with three pairs of lobes. Abbreviations: $\mathrm{AP}=$ apical phallomere; $\mathrm{AS}=$ apical sclerite; $\mathrm{BP}=$ basal phallomere; L 1 = first pair of lobes; L 2 = second pair of lobes; L $3=$ third pair of lobes; MP = median phallomere. 
They are elliptical $(0.58-0.73 \mathrm{~mm} \times 0.40 \mathrm{~mm})$ in shape and are cream or light yellow in color (Fig. 21). Eggs hatched after about 15 days. Grubs are scarabaeiform, C-shaped, dorsum covered with long setae and actively moved forward using the anal pseudopod (Figs 22, 24). Larvae bored into the roots. In the laboratory, they created a tunnel inside pieces of root and stem of seedlings of mango and cashew (Fig. 23). However, none of the larvae survived till the final instar in the laboratory.

\section{Remarks}

The genus in India is represented by two species. Fidia indica (Jacoby, 1908), the other Indian species, can be separated from $F$. kanaraensis by the more regularly arranged elytral punctures (rather confused, with countable rows in posterior half of elytra in F. kanaraensis) and finely transversely wrinkled elytral interstices in the basal area (interstices not wrinkled in F. kanaraensis). Fidia shirozui (Kimoto, 1969), from Taiwan, closely resembles $F$. kanaraensis and differences between these species require additional study.

\section{Discussion}

Fidia kanaraensis, though originally described from southern India, was also recorded in Bengal in eastern India (Jacoby 1908). Such a disjunct distribution is common for many peninsular Indian species

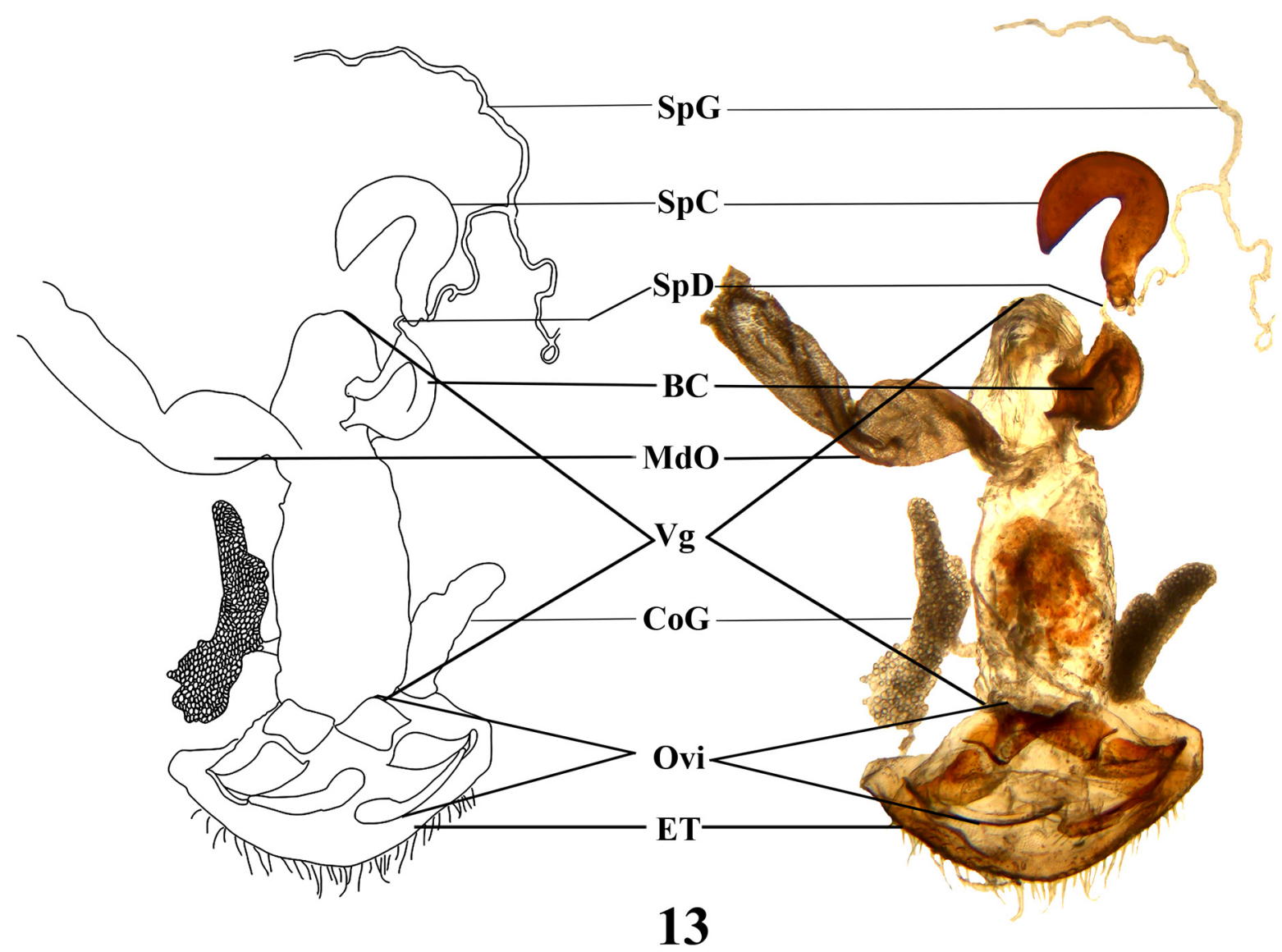

Fig 13. Female genitalia of Fidia kanaraensis (Jacoby, 1895) comb. nov. along with line diagram. Abbreviations: $\mathrm{BC}=$ bursa copulatrix; $\mathrm{CoG}=$ collateral gland; $\mathrm{ET}=$ eighth tergite; $\mathrm{MdO}=$ median oviduct; Ovi $=$ ovipositor; $\mathrm{SpC}=$ spermathecal capsule; $\mathrm{SpD}=$ spermathecal duct; $\mathrm{SpG}=$ spermathecal gland; $\mathrm{Vg}$ = vagina. 

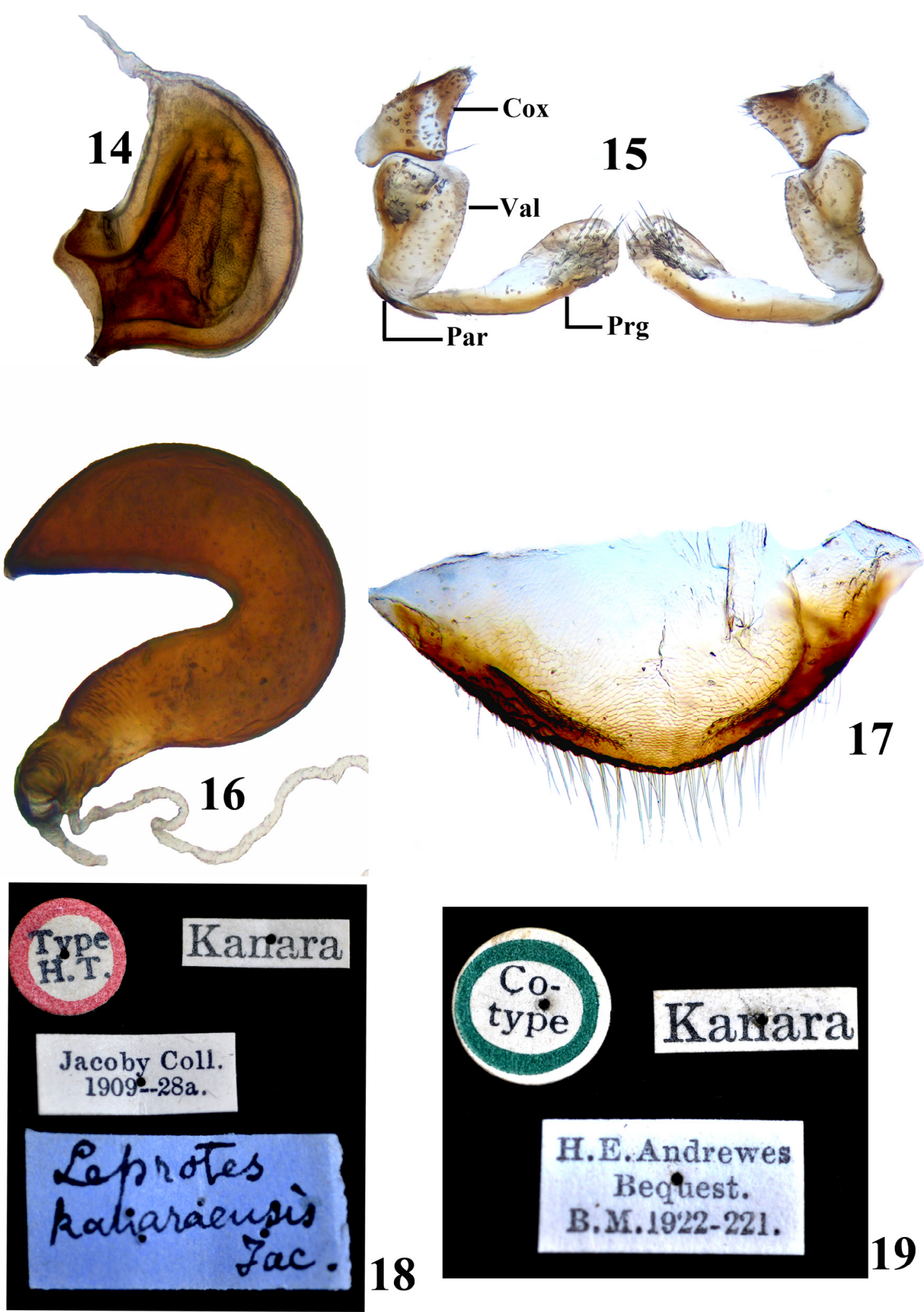

Figs 14-19. Close up of parts of female genitalia of Fidia kanaraensis (Jacoby, 1895) comb. nov. 14. Bursa copulatrix. 15. Ovipositor. 16. Spermatheca. 17. Eighth tergite. 18. Holotype labels. 19. Paratype labels. Abbreviations: $\mathrm{Cox}=$ coxite Par $=$ paraproct Prg $=$ proctiger; Val $=$ valvifer. 
(Hora 1953). The body of $F$. kanaraensis is covered with a white, waxy secretion. This is also known in F. atra, the type species (Chûjô 1954), F. japonicus Ohno, 1958 (= F. babai) (Chûjô 1958b) and F. shirozui Kimoto, 1969. In the Oriental tortoise beetle, Silana farinosa (Boheman), the dorsum is

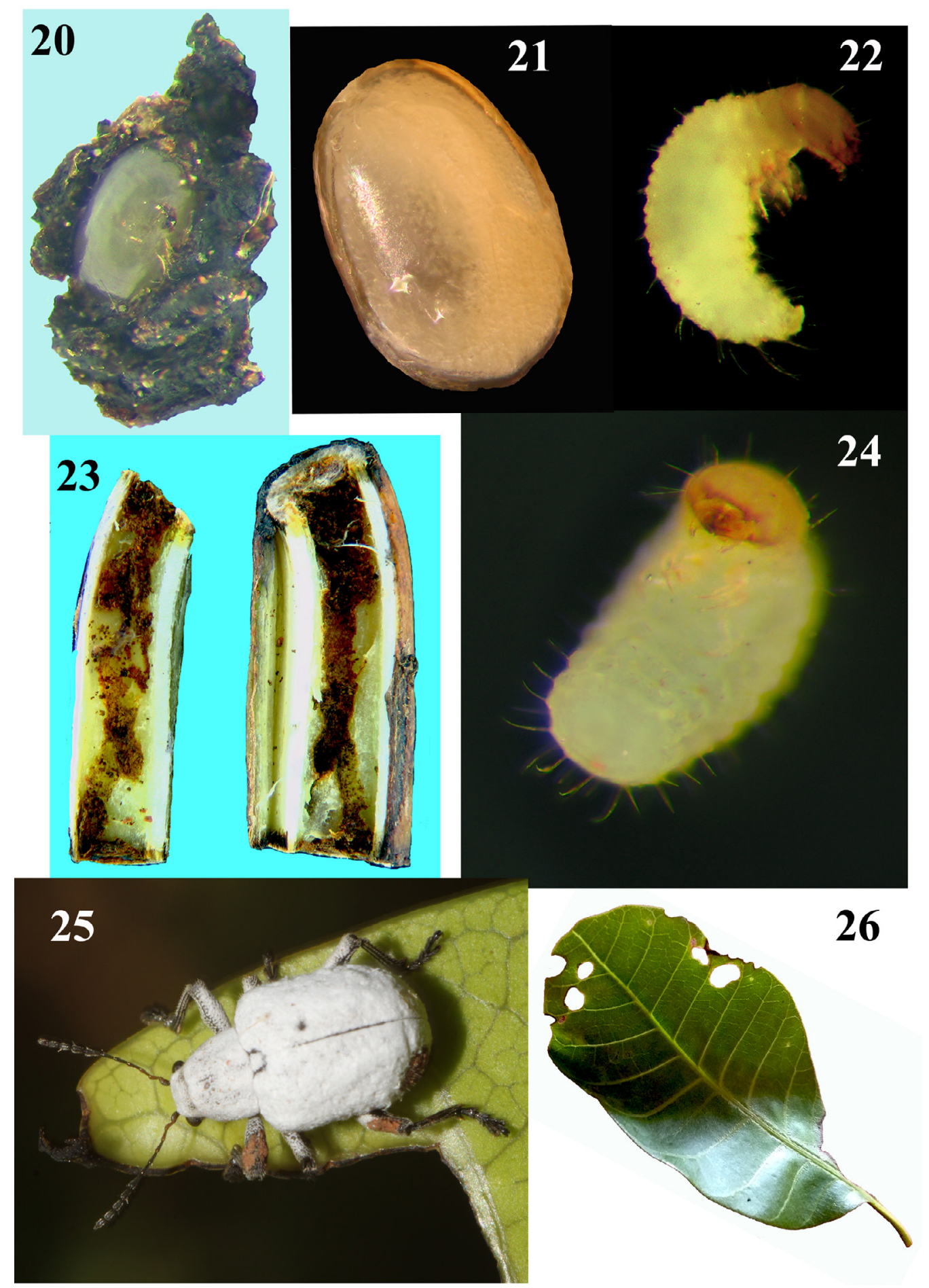

Figs 20-26. Biology and ecology of Fidia kanaraensis (Jacoby, 1895) comb. nov. 20. Eggs covered with excreta. 21. Naked eggs. 22. First instar grub, lateral view. 24. First instar grub, ventral view. 23. Damage on mango root. 25. Adult beetle on mango leaves. 26. Damage on cashew leaves. 
completely covered with a white coating (Maulik 1919). Similarly, in Myrmeconycha Konstantinov \& Tishechkin 2017, a myrmecophilous flea beetle genus from the Neotropics, the head and pronotum are covered with white, waxy exudate. However, the actual origin, chemical nature or function of this white, waxy covering remains unknown.

Flowers (1999), using a different technique for teasing out the endophallus through the apical orifice, studied the endophallus in Eumolpinae and described the structures in the Leprotites. However, in our studies, we have everted the endophallus through the apical opening as well as inflated it (without eversion) through the basal opening. Due to the differing techniques used, comparison of structures with those shown by Flowers (1999) is difficult. A sclerotization on the vaginal wall near the base of the duct is present, as in some other eumolpines (Moseyko 2008; Zoia 2009). The short ovipositor as in Fidia is a progressive character in Chrysomelidae and an adaptation for open oviposition of groups of glued eggs or eggs with some coverage (Moseyko 2008). According to Li \& Liang (2018), females with shorter ovipositors lay eggs on substrate surfaces or in shallow excavations. The female genitalia of F. kanaraensis are different from those of eumolpines such as Platycorynus Chevrolat, 1837 Colasposoma Laporte, 1833 and Chrysochus Chevrolat, 1836, but show a structural similarity to those of Synetinae (Li \& Liang 2018). Treating Synetinae as a tribe within the subfamily Eumolpinae has already been implied by Crowson (1992). The presence of vaginal glands as in Fidia has been reported in Syneta by Reid (1995), who provides evidence strongly in favor of Syneta being a plesiomorphic eumolpine with many adult autapomorphies. However, Verma \& Jolivet (2000) considered Synetinae as a separate subfamily. The pair of vaginal glands (CoG in Fig. 13), observed in F. kanaraensis, are either collateral glands or organs of symbiont transmission (Suzuki 1988). Covering the eggs with excreta and soil along with a glandular secretion (Jolivet et al. 2014) may be a means of transmission of symbionts. Trophic selections of the genus are distributed across plant families (Table 1). However, F. kanaraensis is the only species that feeds on Anacardiaceae. More information on the subterranean immatures of the species is necessary to formulate a management strategy in agro-ecosystems.

\section{Acknowledgments}

We are indebted to Michael Geiser, Natural History Museum, London for the loan of the types and to Dr C.A. Viraktamath, University of Agricultural Sciences, Bangalore for the loan of specimens. The study of A.G. Moseyko was performed based on the ZIN collection within the framework of the state project no. AAAA-A19-119020690082-8 and financially supported by the Russian Foundation for Basic Research (grant no. 19-04-00565 A). SAK is extremely grateful to S.R. Hiremath for helping in collecting specimens, standardizing endophallus inflation technique and his untiring support throughout the work. SAK is indebted to the Kerala Agricultural University for the KAU Fellowship.

\section{References}

Arnett R.H. 1963. The Beetles of the United States. Fascicle 104. Chrysomelidae. Catholic University of America Press, Washington, DC.

Arnett R.H. 1985. American Insects, a Handbook of the Insects of America North of Mexico. Van Nostrand Reinhold Inc, New York.

Balsbaugh E.U. \& Hays K.L. 1972. The leaf beetles of Alabama. Auburn Agricultural Experiment Station Bulletin 441: 1-223.

Baly J.S. 1861. Descriptions of new genera and species of Phytophaga. Journal of Entomology Descriptive and Geographical 1: 275-302.

Baly J.S. 1863. An attempt at a classification of the Eumolpidae. Journal of Entomology, Descriptive and Geographical 2 (9): 143-163. 
Baly J.S. 1878. Description of new species and genera of Eumolpidae. The Journal of the Linnean Society 14: 246-265.

Bechyné J. 1950. Les générotypes des Eumolpides de l'Amérique du Sud et du Centre avec les diagnoses des formes nouvelles (Col. Phytoph. Chrysomeloidea). Mitteilungen der Münchner Entomologischen Gesellschaft 40: 264-292.

Bechyné J. 1953. Katalog der neotropischen Eumolpiden (Col. Phytoph. Chrysomeloidea). Entomologische Arbeiten aus dem Museum G. Frey 4: 26-303.

Blackwelder R.E. 1946. Checklist of the coleopterous insects of Mexico, Central America, the West Indies, and South America. United States National Museum Bulletin 185 (Parts 1-6): 55-763.

Blatchley W.S. 1910. An Illustrated Descriptive Catalogue of the Coleoptera or Beetles (Exclusive of the Rhynchophora) known to occur in Indiana. Nature Publishing Co., Indianapolis.

Chapuis F. 1874. Famille des Phytophages. Vol. 10. In: Lacordaire T. \& Chapuis F. (eds) Histoire naturelle des Insects, Genera des Coléoptères. Roret, Paris.

Chen S.H. 1935. Study on the Chinese eumolpid beetles. Sinensia 6 (3): 221-387.

Chen S.H. 1940. Notes on Chinese Eumolpidae. Sinensia 11 (5-6): 483-528.

Chûjô M. 1935. Chrysomelidae of Loo-Choo Archipelago II. Transactions of Natural History Society of Formosa 25 (142): 203-211.

Chûjô M. 1938. H. Sauter's Formosa Collection: Subfamily Eumolpinae. Arbeiten über morphologische und taxonomische Entomologie aus Berlin-Dahlem 5 (1): 25-36.

Chûjô M. 1954. Descriptions of a new species and a new subspecies of the genus Lypesthes Baly from Japan, including some notice on its own known species (Coleoptera: Chrysomelidae, Eumolpinae). Insecta Matsumurana 18 (3-4): 103-108.

Chûjô M. 1956. A taxonomic study on the Chrysomelidae (Insecta: Coleoptera) from Formosa Part VIII: Subfamily Eumolpinae. The Philippine Journal of Science 85 (1): 1-180.

Chûjô M. 1958a. Chrysomelid-beetles of Loo-Choo Archipelago (V). Memoirs of the Faculty of Liberal Arts \& Education, Kagawa University 2 (64): 1-19.

Chûjô M. 1958b. Chrysomelid beetles from the islands of Iki and Tsushima, Japan, collected by Dr. Baba in 1957. Memoirs of the Faculty of Liberal Arts \& Education, Kagawa University 2 (58): 1-10.

Chûjô M. \& Kimoto S. 1961. Systematic catalogue of Japanese Chrysomelidae (Coleoptera). Pacific Insects 3 (1): 117-202.

Ciegler J.C. 2007. Leaf and Seed Beetles of South Carolina (Coleoptera: Chrysomelidae and Orsodacnidae). Vol. 4: Biota of South Carolina. Clemson University Public service Publishing, Clemson, $\mathrm{SC}$.

Clavareau H. 1914. Chrysomelidae: 11. Eumolpinae pars 59. In: Schenkling S. (ed.) Coleopterorum Catalogus: 1-215. W. Junk. Berlin.

Crotch G.R. 1873. Materials for the study of the Phytophaga of the United States. Proceedings of the Academy of Natural Sciences of Philadelphia 25: 19-83.

Crowson R.A. 1992. Sternal articulations of the mesocoxae in Chrysomelidae. Chrysomela 27: 3.

Dejean P.F.M.A. 1836. Catalogue des Coléoptères de la collection de M. le comte Dejean. MéquiguonMarvis père et fils, Paris. 
Dejean P.F.M.A. 1837. Catalogue des Coléoptères de la collection de M. le comte Dejean [4 ${ }^{\text {th }}$ Ed.]. Méquiguon-Marvis père et fils, Paris.

Eroshkina G.A. 1992. A revision of the genus Lypesthes (Coleoptera, Chrysomelidae, Eumolpinae) of the Vietnam fauna. Sistematika i ekologiya nasekomykh Vietnama [Systematics and Ecology of Insects of Vietnam]: 82-90.

Fairmaire L. 1889. Coléoptéres de l'intérieur de la Chine, $5^{\mathrm{e}}$ partie. Annales de la Société entomologique de France sér. 6, 9: 5-84.

Flowers R.W. 1996. The subfamily Eumolpinae (Coleoptera: Chrysomelidae) in Central America. Instituto Nacional de Biodiversidad, Costa Rica.

Flowers R.W. 1999. Internal structure and phylogenetic importance of male genitalia in Eumolpinae. In: Cox M.L. (ed.) Advances in Chrysomelidae Biology 1: 71-93. Backhuys Publishers, Leiden, Netherlands.

Gemminger M. \& von Harold B. 1874. Catalogus Coleopterum hucusque descriptorum, synonymicus et systematicus. Tome XL Chrysomelidae (Pars I). Williams and Norgate, London.

https://doi.org/10.5962/bhl.title.9089

Gistel J. 1848. Naturgeschichte des Thierreichs für höhere Schulen. Scheitlin und Krais, Stuttgart.

Gistel J. 1851 Naturgeschichte des Thierreichs für höhere Schulen $\left[2^{\text {nd }}\right.$ ed.]. Scheitlin und Krais, Stuttgart. https://doi.org/10.5962/bhl.title.97235

Glover T. 1868. Report of the entomologist. In: Stokes J.W. (ed.) Report of the Commissioner of Agriculture for the year 1867: 58-76. Government Printing Office, Washington D.C.

Gressitt J.L. \& Kimoto S. 1961. The Chrysomelidae (Coleopt.) of China and Korea, part 1. Pacific Insects Monograph 1A: 1- 299.

Griffin F.J. 1936. XXI. On the dates of publication of Motschulsky (V. de), 'Etudes Entomologiques', I-XI, 1853-1862. Annals and Magazine of Natural History 17: 256-257.

https://doi.org/10.1080/00222933608655117

Henshaw S. 1885. List of the Coleoptera of America, North of Mexico. American Entomological Society, Philadelphia. https://doi.org/10.5962/bhl.title.13151

Hora S.L. 1953. The Satpura Hypothesis. Science Progress (1933-) 41 (162): 245-255.

Horn G.H. 1892. The Eumolpini of Boreal America. Transactions of the American Entomological Society 19: 195-234.

International Commission on Zoological Nomenclature (ICZN). 2009. Opinion 2227 (Case 3375) Fidia Baly, 1863 and Lypesthes Baly, 1863 (lnsecta, Coleoptera): usage not conserved and priority maintained for Fidia Motschulsky, 1860. Bulletin of Zoological Nomenclature 66 (2): 198-200.

https://doi.org/10.21805/bzn.v66i2.a12

Isono M. 1988. Differentiation in life history pattern and oviposition behavior, and thelytoky in Demotina and Hyperaxis beetles (Coleoptera, Chrysomelidae) in western Japan. Kontyu, Tokyo 56 (2): 402-409.

Jacoby M. 1879. Descriptions of new species of phytophagous Coleoptera. Proceedings of the Scientific Meetings of the Zoological Society of London 47 (1): 773-793.

https://doi.org/10.1111/j.1096-3642.1879.tb02716.x

Jacoby M. 1882. Insecta. Coleoptera (vol VI Part I) Phytophaga (part). Biologia Centrali-Americana 6 (1): 1-625. https://doi.org/10.5962/bhl.title.730 
Jacoby M. 1885. Descriptions of the phytophagous Coleoptera of Japan, obtained by Mr. George Lewis during his second journey, from February 1880 to September 1881.- Part I. Proceedings of the Scientific Meetings of the Zoological Society of London 53 (1): 190-211.

https://doi.org/10.1111/j.1096-3642.1885.tb02894.x

Jacoby M. 1890. Descriptions of new species of phytophagous Coleoptera received by Mr. J.H. Leech, from Chang-Yang, China. The Entomologist, An Illustrated Journal of General Entomology 23: 114118.

Jacoby M. 1895. Descriptions of the new species of phytophagous Coleoptera obtained by M. Andrewes in India. Annales de la Société entomologique de Belgique 39: 252-288.

Jacoby M. 1908. The Fauna of British India, including Ceylon and Burma. Coleoptera. Chrysomelidae, Vol. 1. Taylor \& Francis, London. https://doi.org/10.5962/bhl.title.48423

Jolivet P. \& Hawkeswood T.J. 1995. Host Plants of Chrysomelidae of the World. Backhuys Publishers, Leiden, Netherlands.

Jolivet P. \& Verma K.K. 2008. Eumolpinae-a widely distributed and much diversified subfamily of leaf beetles (Coleoptera, Chrysomelidae). Terrestrial Arthropod Reviews 1: 3-37.

https://doi.org/10.1163/187498308X345424

Jolivet P., Lawrence J.F., Verma K.K. \& Ślipiński A. 2014. 2.7.3 Eumolpinae C.G. Thompson, 1859. In: Leschen R.A.B. \& Beutel R.G. (eds) Handbook of Zoology, Arthropoda: Insecta, Coleoptera (Beetles), Vol. 3: Morphology and Systematics (Phytophaga): 217-225. De Gruyter.

https://doi.org/10.1515/9783110274462

Kimoto S. 1964. The Chrysomelidae of Japan and the Ryukyu Islands. IV. Journal of faculty of Agriculture, Kyushu University 13 (2): 235-262.

Kimoto S. 1969. Notes on Chrysomelidae from Taiwan II. Esakia 7: 1-68.

Kimoto S. 2005. Systematic catalogue of Chrysomelidae from Nepal and Bhutan. Bulletin of Kitakyushu Museum of Natural History and Human History Series A 3: 1-180.

Kimoto S. \& Gressitt J.L. 1966. The Chrysomelidae of Ryukyu Archipelago. Pacific Insects 8 (2): 467-577.

Kimoto S. \& Gressitt J.L. 1982. Chrysomelidae (Coleoptera) of Thailand, Cambodia, Laos and Vietnam. III. Eumolpinae. Esakia 18: 1-141.

Konstantinov A.S. 1998. On the structure and function of the female genitalia in flea beetles (Coleoptera: Chrysomelidae: Alticinae). Proceedings of Entomological Society of Washington 100 (2): 353-360.

Lee J.N. 2012. Leaf beetles (Larvae). Arthropoda: Insecta: Coleoptera: Chrysomelidae (larvae). 12 (6): Insect Fauna of Korea. National Institute of Biological Resources, Incheon, Korea.

Lefèvre E. 1877. Descriptions de Coléoptères nouveaux ou peu connus de la Famille des Eumolpides ( $1^{\text {re }}$ partie). Annales de la Société entomologique de France sér. 5, 7: 115-166.

Lefèvre E. 1885. Eumolpidarum hucusque cognitarum catalogus, sectionaum conspectus systematico, generum sicut et specierum nonnullarum novarum descriptionibus adjunctus. Mémoires de la Société Royal des Sciences de Liège 11: 3-172.

Leng C.W. 1920. Catalogue of the Coleoptera of America, North of Mexico. J.D. Sherman, Jr. Mount Vernon, N.Y.

LeSage L. 1991. Family Chrysomelidae, leaf beetles. In: Bousquet Y. (ed.) Checklist of beetles of Canada and Alaska: 301-323. Agriculture Canada Publication 1861E. 
Li K. \& Liang H. 2018. A comparative study of external genitalia (including the $8^{\text {th }}$ and $9^{\text {th }}$ abdominal segments) in the family Megalopodidae and other related families of Chrysomeloidea. Zookeys 762 : 69-104. https://doi.org/10.3897/zookeys.762.22163

Löbl I. 2010. Chrysomelidae: Eumolpinae (New acts and comments). In: Löbl I. \& Smetana A. (eds) Catalogue of Palaearctic Coleoptera. Vol. 6. Chrysomeloidea: 83. Apollo Books, Stenstrup.

Maulik S. 1919. The fauna of British India, including Ceylon and Burma. Coleoptera. Chrysomelidae (Hispinae and Cassidinae). Taylor \& Francis, London.

Medvedev L.N. 1993. New species of Chrysomelidae from South Asia. Entomologica Basiliensia 16: 359-376.

Medvedev L.N. 2007. New and poorly known Oriental Chrysomelidae (Coleoptera) of the Staatliches Museum fur Naturkunde, Stuttgart. Stuttgarter Beiträge zur Naturkunde Serie A (Biologie) 702: 1-19.

Medvedev L.N. 2015. New and poorly known Oriental Chrysomelidae (Insecta: Coleoptera) in the collection of the Naturkundemuseum Erfurt. Vernate 34: 319-335.

Medvedev L.N. \& Zoia S. 1996. New and interesting species of Eumolpinae from Pakistan and Thailand (Coleoptera Chrysomelidae). Bollettino di Zoologia Agraria e Bachicoltura 28 (2): 111-117.

Melsheimer F.E. 1847. Descriptions of new species of Coleoptera of the United States. Proceedings of the Academy of Natural Sciences of Philadelphia 3 (1): 158-181.

Moseyko A.G. 2008. The Reproductive Apparatus of Female Leaf Beetles (Coleoptera, Chrysomelidae) and its Importance for the Systematics of the Group. PhD thesis, Russian Academy of Sciences, Zoological Institute.

Moseyko A.G. \& Kirejtshuk A.G. 2013. On the taxonomic position of new taxa of the subfamily Eumolpinae (Coleoptera, Chrysomelidae) from Baltic amber. Terrestrial Arthropod Reviews 6: 163-172. https://doi.org/10.1163/18749836-06021063

Moseyko A.G. \& Sprecher-Uebersax E. 2010. Subfamily Eumolpinae Hope, 1840. In: Löbl I. \& Smetana A. (eds) Catalogue of Palaearctic Coleoptera. Vol. 6: Chrysomeloidea: 619-643. Apollo Books, Stenstrup.

Motschulsky V. 1861. Insectes du Japon. Études entomologiques 9: 4-39.

Ohno M. 1958. On the genus Lypesthes Baly from Japan (Coleoptera: Chrysomelidae, Eumolpinae). Journal of the Toyo University 12: 173-181.

Pic M. 1923. Nouveautés diverses. Mélanges exotico-entomologiques 40: 1-32.

Pic M. 1924. Nouveautés diverses. Mélanges exotico-entomologiques 41: 1-32.

Pic M. 1928. Nouveautés diverses. Mélanges exotico-entomologiques 52: 1-32.

Pic M. 1936. Nouveautés diverses. Mélanges exotico-entomologiques 67: 1-32.

Pic M. 1939. Nouveaux coléoptères, principalement phytophages, de l'Ethiopie et Somalie italienne. Memorie della Società Entomologica Italiana 17 (1): 31-37.

Rajendran T.P. \& Singh D. 2016. Chapter 1. Insects and pests. In: Omkar (ed.) Ecofriendly Pest Management for Food Security: 1-24. Academic Press, UK.

https://doi.org/10.1016/B978-0-12-803265-7.00001-4

Reid C.A.M. 1995. A cladistic analysis of subfamilial relationships in the Chrysomelidae sensu lato (Chrysomeloidea). In: Pakaluk J. \& Ślipiński S.A. (eds) Biology, Phylogeny, and Classification of Coleoptera Papers Celebrating the 80 ${ }^{\text {th }}$ Birthday of Roy A. Crowson: 559-631.Warzawa Muzeum i Instytut Zoologii PAN. 
Riley E.G., Clark S.M., Flowers R.W. \& Gilbert A.J. 2002. 124. Chrysomelidae Latreille 1802. In: Arnett R.H., Thomas M.C., Skelley P.E. \& Frank J.H. (eds) American Beetles. Vol. 2, Polyphaga: Scarabaeoidea through Curculionoidea: 617-691. CRC Press. New York.

Riley E.G., Clark S.M. \& Seeno T.N. 2003. Catalog of the leaf beetles of America north of Mexico (Coleoptera: Megalopodidae, Orsodacnidae and Chrysomelidae, excluding Bruchinae). Coleopterists Society Special Publication No.1.

Schaeffer C. 1904. New genera and species of Coleoptera Class I, Hexapoda Order II, Coleoptera. Journal of the New York Entomological Society 12 (4): 197-236.

Schaeffer C. 1933. Short studies in the Chrysomelidae (Coleoptera). Journal of the New York Entomological Society 41: 457-480.

Seeno T.N. \& Wilcox J.A. 1982. Leaf Beetle Genera (Coleoptera: Chrysomelidae). Entomography Publications, California.

Ślipiński A. \& Escalona H.E. 2013. Australian Longhorn Beetles (Coleoptera: Cerambycidae). Vol. 1. CSIRO Publishing, Melbourne.

Strother S.M. 2003. Genus Fidia Baly. In: Riley E.G., Clark S.M. \& Seeno T.N. (eds) Catalog of the Leaf Beetles of America North of Mexico (Coleoptera: Megalopodidae, Orsodacnidae and Chrysomelidae, excluding Bruchinae): 151-152 \& 192-193. Coleopterists Society.

Strother M.S. \& Staines C.L. 2008. A revision of the New World genus Fidia Baly, 1863 (Coleoptera: Chrysomelidae: Eumolpinae:Adoxini). Zootaxa 1798: 1-100. https://doi.org/10.11646/zootaxa.1798.1.1

Strother M.S, Bayless V.M. \& Staines C.L. 2007. Case 3375 Fidia Baly, 1863 and Lypesthes Baly, 1863 (Insecta, Coleoptera): proposed conservation of usage. Bulletin of Zoological Nomenclature 64: 35-38.

Sturm J. 1826. Catalog meiner Insecten-Sammlung von Jacob Sturm. Erster Theil Käfer. Privately published, Nürnberg. https://doi.org/10.5962/bhl.title.15090

Sturm J. 1843. Catalog der Kaefer-Sammlung von Jacob Sturm. Privately published, Nürnberg. https://doi.org/10.5962/bhl.title.37837

Suzuki K. 1988. 19. Comparative morphology of the internal reproductive system of the Chrysomelidae (Coleoptera). In: Jolivet P., Petitpierre E. \& Hsiao T.H. (eds) Biology of Chrysomelidae: 317-342. Kluwer Academic Publishers, Netherlands.

Tan J. 1983. On a newly recorded genus and two new species of Eumolpidae. Wuyi Science Journal 3: $129-130$.

Uhler P.R. 1855. Descriptions of a few species of Coleoptera, supposed to be new. Proceedings of the Academy of Natural Sciences in Philadelphia 7: 415-418.

Verma K.K. \& Jolivet P. 2000. Phylogeny of Synetinae-reconsidered. Nouvelle Revue d'Entomologie (N.S.) 17 (1): 35-49.

Walsh B.D. 1867. The grape-vine Fidia (Fidia viticida, new species). Practical Entomologist 2 (8): $87-88$.

Wilcox J.A. 1954. Leaf beetles of Ohio (Chrysomelidae: Coleoptera). Ohio Biological Survey Bulletin 43: 353-506.

Wilcox J.A. 1975. Checklist of the Beetles of Canada, United States, Mexico, Central America, and the West Indies 1 (7): The Leaf Beetles (red version). Biological Research Institute of America, Latham.

Williston S.W. 1889. Notes on Asilidae. Psyche, A Journal of Entomology 5 (160-164): 255-259. 
Yamasako J. \& Ohbayashi N. 2011. Review of the genus Paragolsinda Breuning, 1956 (Coleoptera, Cerambycidae, Lamiinae, Mesosini), with reconsideration of the endophallic terminology. Zootaxa 2882: 35-50. https://doi.org/10.11646/zootaxa.2882.1.4

Zhou H. \& Tan J. 1997. Faunistic notes and three new species of Eumolpinae from Fujian province, China (Coleoptera). Acta Zootaxonomica Sinica 22 (2): 189-193.

Zoia S. 2009. A revision of the genus Mecistes (Coleoptera Chrysomelidae). Memorie della Società entomologica italiana 88: 117-154.

Manuscript received: 5 September 2019

Manuscript accepted: 14 April 2020

Published on: 28 May 2020

Topic editor: Gavin Broad

Section editor: Max Barclay

Desk editor: Pepe Fernández

Printed versions of all papers are also deposited in the libraries of the institutes that are members of the EJT consortium: Muséum national d'histoire naturelle, Paris, France; Meise Botanic Garden, Belgium; Royal Museum for Central Africa, Tervuren, Belgium; Royal Belgian Institute of Natural Sciences, Brussels, Belgium; Natural History Museum of Denmark, Copenhagen, Denmark; Naturalis Biodiversity Center, Leiden, the Netherlands; Museo Nacional de Ciencias Naturales-CSIC, Madrid, Spain; Real Jardín Botánico de Madrid CSIC, Spain; Zoological Research Museum Alexander Koenig, Bonn, Germany; National Museum, Prague, Czech Republic. 